\title{
Research on glass wall cleaning robot based on double suction cup negative pressure type
}

\author{
Wu Guifang ${ }^{1,2, a}$, Zhang Bowei ${ }^{2}$, Qi Panpan², Shao Cai ${ }^{2}$, Guo Yongzhen² \\ ${ }^{1}$ Control science and engineering postdoctoral research station, Henan Univ. of Sci. \& Tech., \\ Luoyang 471000, China \\ ${ }^{2}$ Information Engineering College, Henan Univ. of Sci. \& Tech., Luoyang 471000, China \\ aeasyfancy@126.com
}

Keywords: Wall robot, negative pressure suction, sliding friction, internal air flow field.

\begin{abstract}
With the rapid development of society, improve space utilization, modern city high-rise building height are also increasing rapidly, the glass wall clean, damage detection, equipment maintenance and other aspects of the problems keep cropping up. Using robots to replace human tasks not only reduces the risk and cost of operation, but also improves the efficiency of the operation. Negative pressure type double suction cup wall robot in the process of sports remain the state of sliding friction between sucker and metope, this requires that chuck has good abrasion resistance and sliding friction coefficient between the walls is small, looking for the right sucker material becomes the key. Based on the study of materials, structural mechanics to design a kind of negative pressure type double suction cup sealing mechanism, reduce the suction cup cavity air leakage, ensure the safety of robot adsorption at the same time, and improve the performance of wall adaptability and locomotion. By the wall robot adsorption method and control technology research, and according to the faults of big friction resistance between sliding type robot sucker and the wall and inflexible movement, a negative pressure adsorption double suction cup sealing mechanism is designed, and a wall robot principle prototype is produced, and the feasibility of the method is validated by experiments. And at the same time, system intelligent algorithms are researched and realized, in order to assure the robot's stable operation.
\end{abstract}

\section{Introduction}

Wall robot is a mechanical structure design, sensor, electronics, control and information technology as one of the high-tech products, and is a kind of robot that can work in either a vertical or inclined relative movement on a smooth surface. From last century, There are many research in the field of wall climbing robot, especially in Japan, U.S., Russia etc., but there are kinds of different problems which cause the robot hard to be used. For example, in 1966, the world's first mobile robot principle prototype is produced in Japan, and after several years, a "walker" robots is produced, in 2008, Rentschler Andrew J et al. performed clinical evaluation on walking robots and achieved certain results. Ultrastrip corporation of U.S. in 2010 developed a single sucker wheel spray painting robot. In China, the research is much late, but there are many universities and research institutions input into the research, such as Harbin institute of technology, Beijing university of aeronautics and astronautics, Tsinghua University, etc., and a lot has been achieved in wall climbing robot, especially in single suction cup wall robot. But for this single suction cup wall robot, it is simple in terms of machinery and control, and it requires wall certain smoothness, and the obstacle ability is poor, it is hard for the complicated surface environment with larger grooves, concaves and convexes, suction cups in the negative pressure is difficult to maintain, and the suction cup sealing edge wear because of the relative sliding.

\section{Overall system structure design}


The overall design of the robot is shown in figure 1, with the ATMEGA128A chip as the core of the overall design framework. The design system consists of power module, wheel motor drive module, negative pressure adsorption module and edge detection module etc.

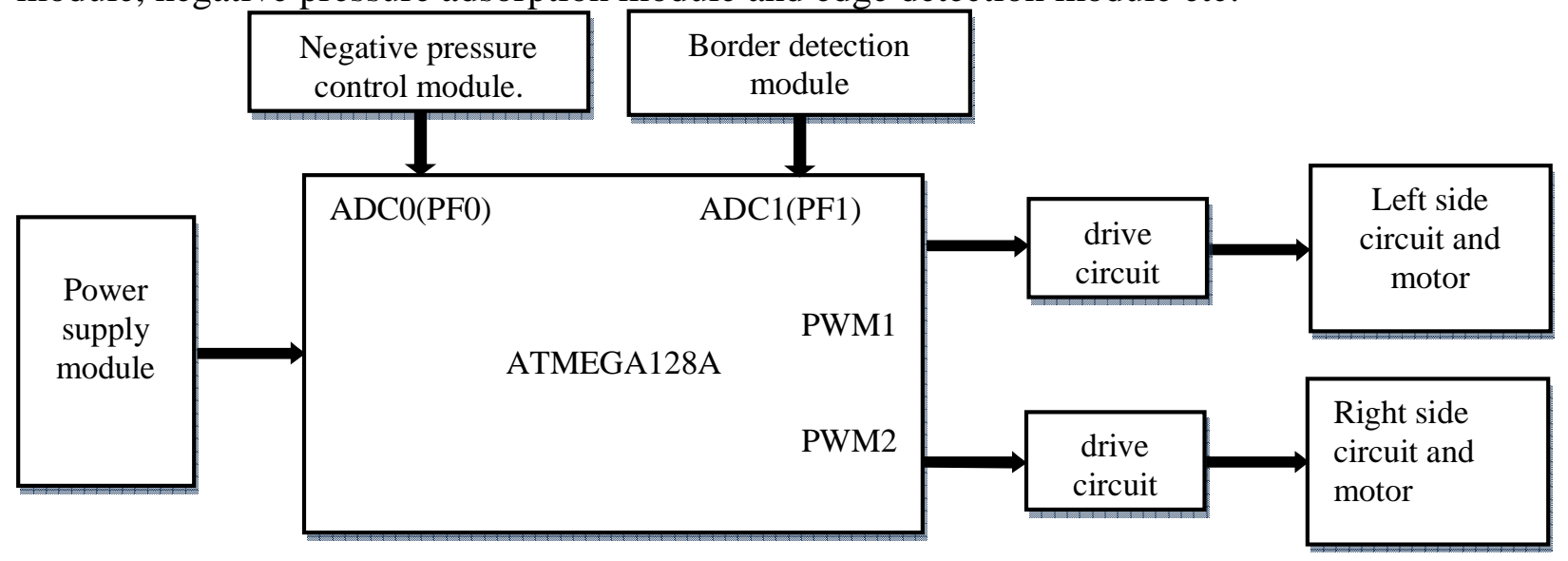

Fig. 1 Overall functional structure block diagram.

\section{Research and design of system intelligent algorithm}

The robot's working process is divided into three parts: self-inspection, initialization and operation. Self- inspection and initialization process to ensure that mechanical devices work properly. The robot has two kinds of manual mode and automatic mode, which can choose the working mode of robot according to its own needs. Autonomous path planning, scheduling and driving can be realized under automatic robot mode. First, the robot will be scrubbed according to its internal memory path, and will adjust its direction when touching the border. Next, a reasonable motion planning scheme is formed to allocate, execute and manage the sequence of actions for specific tasks, and form various status symbols; In manual mode, select the position of scrubbing and the number of scrubbing and the direction of the movement according to the control of the operator's remote control. The general flow chart of the robot's software system algorithm is shown in figure 2.

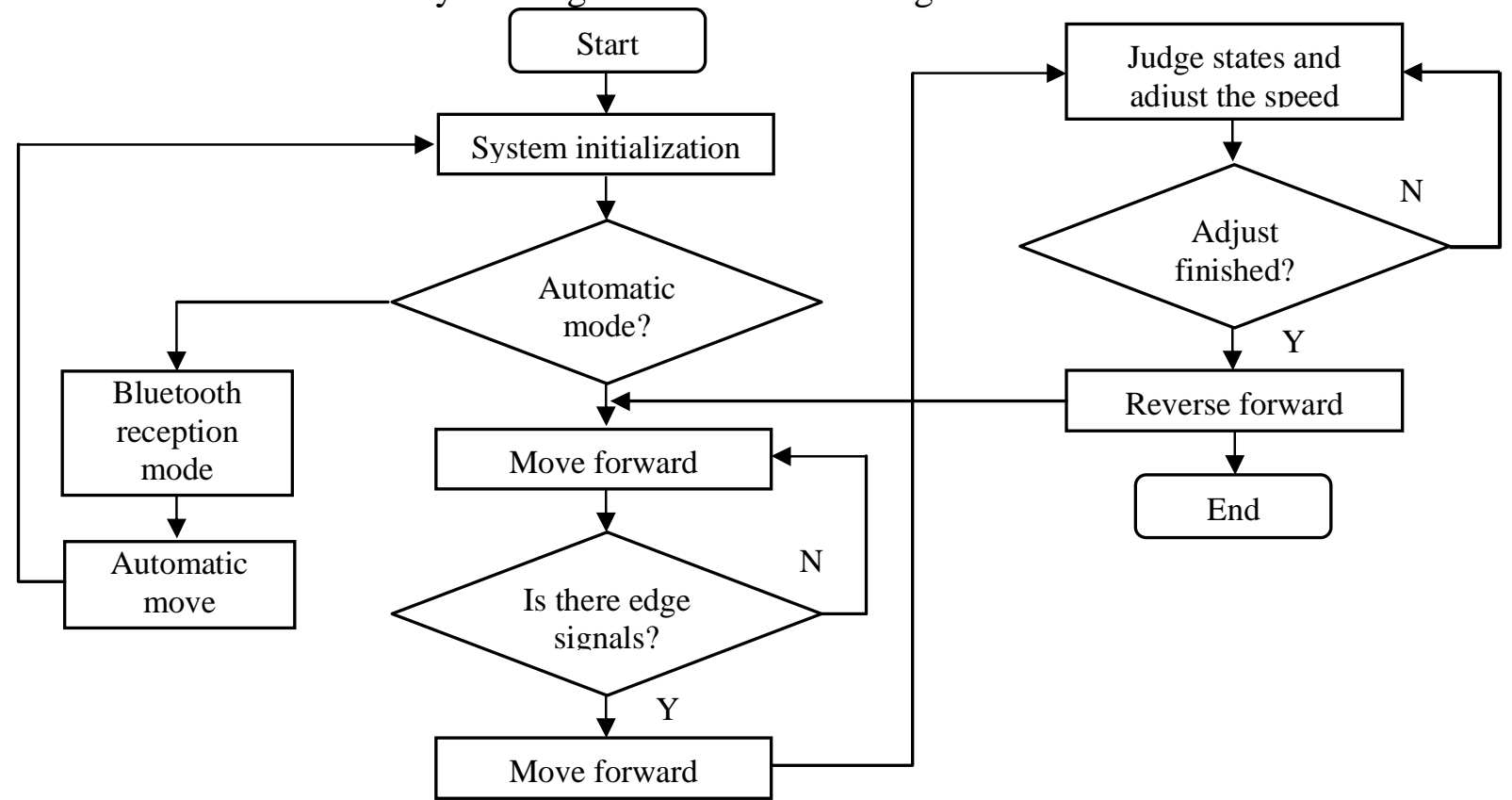

Fig.2. General flow chart of software system algorithm.

The control system is the core component of the glass wall cleaning robot, which is responsible for the core functions such as the adsorption and driving of the glass wall robot. Its basic requirements are convenient, reliable and flexible. The control system consists of two parts: negative pressure control system and motion control system. The control of negative pressure is the foundation of 
whether the glass wall robot can work normally, and the motion control mainly completes the motion function of the robot on the wall surface. Flow chart of robot control algorithm is shown in figure 3.

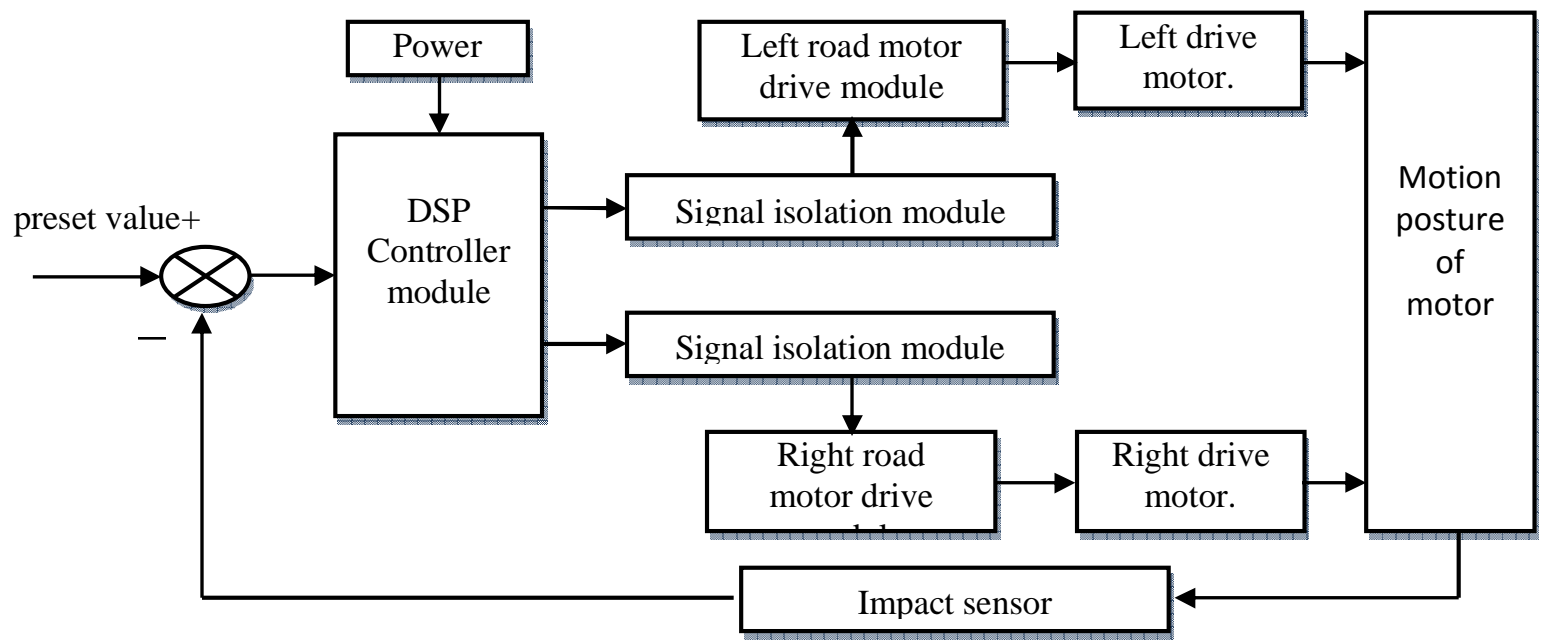

Fig.3 Control system algorithm flow chart.

Adopted due to the robot motion and structure of integration design scheme, at the same time of movement to complete the work of cleaning the glass wall, so the robot's path planning software planning including operations and motion planning of two parts. The task path planning is used to complete the planning and scheduling of the cleaning sequence and steps of the entire glass wall surface. The action plan is used to select the sequence of actions required to complete the task, and the reasonable cooperation between them ensures the smooth completion of the wall cleaning robot operation. The software architecture of robot mobile module system is shown in figure 4.

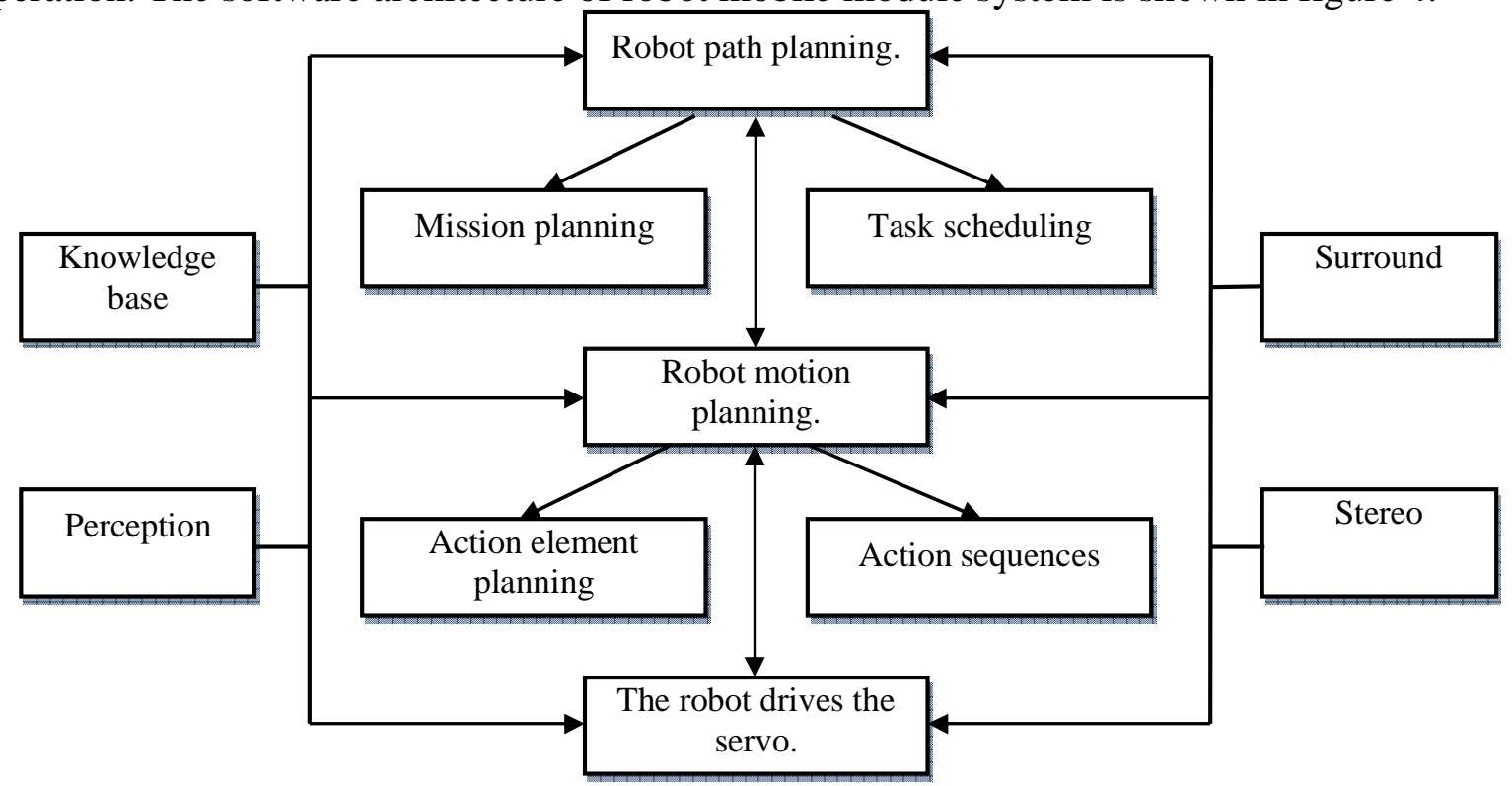

Fig.4 Robot mobile module algorithm flow chart.

In order to achieve the close adsorption, the adsorption device adopts the circular suction cup and the small vacuum pump composite structure. The sliding friction force of the suction cup is small, the sealing effect is good, and the sliding suction cup is produced by the micro-centrifugal vacuum pump to produce negative pressure in the sealing chamber of the sucker, which is closely adsorbed on the glass wall surface. At the same time, the adsorption of adsorption device adopts two child chucks structure, each child chuck with a suction cup by the respective valve control, when any child chuck leakage, the valve can be adjusted in a timely manner to control the negative pressure value, and can use the suction cup deformation of elastic skirt to adapt to the uneven surface, make sure reliable sealing sucker and the wall. Therefore, the structure of the multipliers allows the suckers to maintain a balance when they encounter grooves and concave surfaces. The structure is shown as Figure 5. 


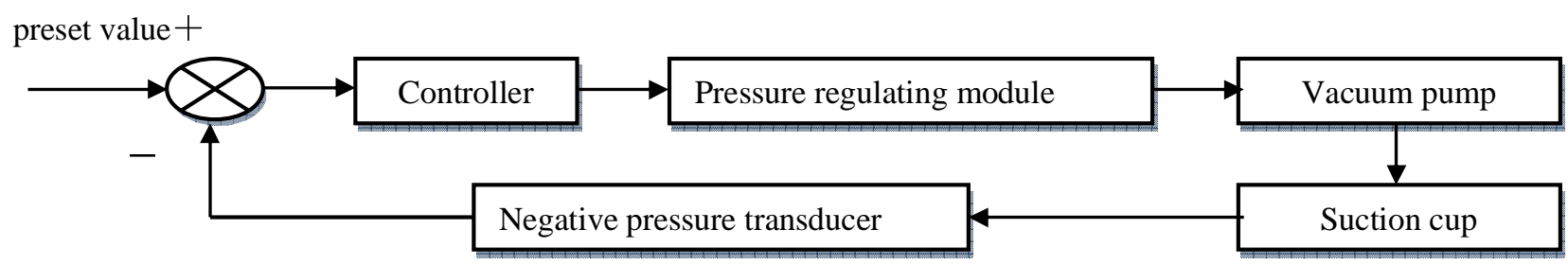

Fig.5 Functional block diagram of adsorption system structure.

\section{Conclusions}

Because the single suction cup glass wall robot is simple in terms of machinery and control, and it requires wall certain smoothness, and its obstacle ability is poor, it is hard to adapt complicated surface with larger grooves. A glass wall cleaning robot based on double suction cup negative pressure type is researched, and by researching fluid modeling analysis of the flow field inside the suction cup mathematical theory, a kind of negative pressure double suction cup sealing mechanism of adsorption is designed, including its overall system structure and system intelligent control algorithm. The experiments of the designed glass wall cleaning robot prototype show that the suction seal mechanism designed according to the principle of gas seal can meet the requirements of robot adsorption, and the given control algorithms are fast and stable, and can meet the realization requirement.

\section{Acknowledgements}

This work was financially supported by International Cooperation in Science and Technology Project (2011DFR10480), Talent Science Research Foundation of Henan Univ. of Sci. \& Tech. (No.09001121), Post-doctoral research fund and SRTP of Henan Univ. of Sci. \& Tech.

\section{References}

[1] Rentschler Andrew J, Simpson Richard, Cooper Rory A. Clinical evaluation of Guido robotic walker. Journal of Rehabilitation Research and Development, 2008, 45(9): 1281-1294

[2] Ge Dingxin, Ren Chao, Matsuno Takahiro, etc. Guide rail design for a passive suction cup based wall-climbing robot, IEEE International Conference on Intelligent Robots and Systems, 2016, 5776-5781

[3] Mohamed Ali M., Zyada Zakarya A., El-Shenawy E.A. Design, modeling and control of a wall climbing robot crossingover obstacles. 2014 IEEE/SICE International Symposium on System Integration, 2014: 46-51

[4] Lee Jeesoo, Kim Sanghoon. Suction based wall climbing robot for edge movement. Lecture Notes in Electrical Engineering, 2014, 301: 687-696

[5] Wang Tai-Yong, Xu Ai-Fen, Zhao Li. Safety of wall robot's vacuum system based on redundant control theory. Journal of Tianjin University Science and Technology,2008,41(3): 261-266

[6] Liu Jiajun, Sun Zhenguo, Zhang Wenzeng. Plane-constraint based hand-eye calibration method for underactuated wall-climbing robot. Robot, 2015,37(3): 271-276, 285

[7] Mohamed Ali M, Zyada Zakarya A, El-Shenawy E.A. Design, modeling and control of a wall climbing robot crossingover obstacles. 2014 IEEE/SICE International Symposium on System Integration, SII 2014: 46-51 